Der "Steady-State” als Leitbild einer vorsichtsorientierten Umweltpolitik

\section{Sicherheitsmarge statt Optimum}

\author{
Eine Senkung und Stabilisierung des Material- und Energiedurchsatzes in den \\ OECD-Staafen gilt vielen als notwendige Bedingung einer zukunftsfähigen \\ Entwicklung. Der wichtigste Vertreter dieser Überlegung - die auch als \\ Steady-State-Wirtschaft bezeichnet wird - ist Herman Daly. Wenn die \\ Leitlinien dieses Ansatzes akzeptiert werden, hat dies erhebliche - und nicht \\ unproblematische - Konsequenzen für die Gestaltung der Umweltpolitik.
}

$\mathrm{W}$

Von Fred Luks ann befindet sich eine Wirtschaft im Steady-State? Anfangs rekurrierte Herman Daly auf eine „,bestandsorientierte“ Definition des Steady-State als ,an economy with constant stocks of people and artifacts, maintained at some desired, sufficient levels by low rates of maintenance 'throughput', that is, by the lowest feasible flows of matter and energy from the first stage of production (...) to the last stage of consumption" (1). Seit längerer Zeit findet sich in Dalys Schriften eine ,flußorientierte" Definition, nach der eine Steady-State-Economy eine Wirtschaft ist, ,whose throughput remains constant at a level that neither depletes the environment beyond its regenerative capacity, nor pollutes it beyond its absorptive capacity“ (2). Beide Versionen laufen auf ein und dasselbe Ziel hinaus, nämlich die Stabilisierung des Material- und Energiedurchsatzes der Anthroposphäre, des Throughputs. Den Umfang dieses Throughput bezeichnet Daly auch als Scale oder Ausmaß der Wirtschaft. Eine SteadyState-Economy ist somit eine Wirtschaft, in der der Throughput/Scale konstant ist.

Scale - die absolute physische Größe der Wirtschaft - ist ein Problem, das von der Frage der Allokation abzugrenzen ist (auch wenn hier Interdependenzen bestehen). Daly verdeutlicht den fundamentalen Unterschied zwischen Scale und Allokation mit der folgenden Metapher: ,When the watermark hits the Plimsoll line the boat is full, it has reached its safe carrying capacity. Of course, if the weight is badly allocated, the water line will touch the Plimsoll mark sooner. But eventually as the absolute load is increased, the watermark will reach the Plimsoll line even for a boat whose load is optimally allocated. Optimally loaded boats will sink under too much weight - even though they may sink optimally! It should be clear that optimal allocation and optimal scale are quite distinct problems“ (3). Wenn der Scale heute zu groß ist, muß er reduziert werden, wenn nachhaltige Entwicklung das Ziel ist. Zwischen der Wachstumswirtschaft und dem Steady-State liegt der declining state - eine Reduktion des Scale. Aber um welchen ,Faktor“? Wieviel ist genug?

\section{Wieviel Umwelt braucht die Wirtschaft?}

Wie viele andere WissenschaftlerInnen sieht auch Daly, daß ein Steady-State erst dann Sinn macht, wenn der Throughput ein ,zukunftsfähiges" Niveau erreicht hat. Nun ist es allerdings nicht möglich, einen solchen Level zu bestimmen. Denn ein „sustainable throughput“ wäre einer, der die Tragekapazität der Umwelt nicht übersteigt, und zwar weder auf der Quellennoch auf der Senkenseite. Leider weiß niemand - kann niemand wissen - wo diese Tragekapazität aufhört und wo ökologische Wachstumsgrenzen anfangen. Die Komplexität natürlicher Prozesse macht solche Voraussagen unmöglich. Diese Sachlage ist zwar ein Argument gegen die Vorstellung, einen ,optimalen “ Scale bestimmen zu können, aber gleichzeitig die wichtigste Begründung für eine Politik der Scale-Reduktion, die auf die Senkung des Material- und Energiedurchsatzes gerichtet ist.

Wolfgang Sachs zeigt, daß man mit der BootMetapher ein anderes grundlegendes Problem, nämlich ,den tiefsitzenden Widerstreit der Grundorientierungen im heutigen Umweltdenken“, veranschaulichen kann: „Denn die Besatzung des Schiffes (...) hat zwei Möglichkeiten. Entweder sie versucht, bei jedem Wetter, bei allen Strömungen und bei jedem Wellengang das Schiff so voll wie gerade noch möglich zu packen. Sie wird dafür das Schiff (...) so umbauen, daß das Schiff immer bis haarscharf an der Grenze seiner Tragekapazität bleibt. Oder aber die Besatzung (...) richtet sich auf dem Schiff nach ihrem Gutdünken und ihrer Lebensphilosophie so ein, daß man sich nur am Rande über Belastungsgrenzen $\mathrm{zu}$ kümmern braucht, also weder die ganze Einrichtung auf optimale Nutzung zuschneiden, noch eine Kybernetik der Selbstbeobachtung aufbauen muß" (4). Es spricht einiges dafür, ein Durchsatzniveau anzusteuern, das nicht gerade noch nachhaltig ist, sondern eines, das eine gewisse Sicherheitsmarge berücksichtigt. Warum?

\section{- Leitbild Vorsichtsprinzip}

Die menschlichen Kenntnisse über die Funktionsfähigkeit natürlicher Prozesse sind stets begrenzt, und es ist eben nicht zu erwarten, daß sich dies ändern wird. Ein Vorsichtsprinzip ergibt sich mithin nicht zuletzt aus der Berïcksichtigung von ökologischem Nichtwissen und impliziert letztlich auch eine ,Abkehr von der Illusion des Machbarkeitsglaubens heutiger Politik" (5). Diese Überlegung führt zur Forderung nach einer Politik, die auf die Vermeidung potentieller Schäden gerichtet ist, ergo nicht lediglich die Bekämpfung bereits entstandener Schäden anstrebt. Die übliche Definition des Vorsorgeprinzips geht davon aus, daß Maßnahmen zum Umweltschutz dann gerechtfertigt sind, wenn Unsicherheiten hinsichtlich der Gefährdung bestehen und wenn irreversible Schäden nicht auszuschließen sind. Implizit wird dabei oft angenommen, daß die Unsicherheit ein vorübergehendes Problem sei, das durch Forschungsanstrengungen $\mathrm{zu}$ überwinden ist. Ökologisches Nichtwissen und ökologische Unsicherheit lassen sich jedoch nicht einfach durch mehr Forschung beseitigen, womit die Hoffnung auf eine „Lösung“ der Wissensprobleme unrealistisch wird (6). Dazu kommt, daß das Hase- und Igel-Spiel zwischen immer neuen ökologischen Problemen und gesellschaftlichen Reaktionen darauf zu einer immer höheren Regelungsdichte führt, ohne das Problem an seiner Wurzel zu packen.

Es erscheint also als eine sinnvolle Konkretisierung des Vorsichtsprinzips, einen gewissen Sicherheitsabstand zwischen Belastungsgrenzen und tatsächlicher Nutzung zu halten, und zwar im Hinblick auf dem totalen Material- und Energiedurchsatz einer Wirtschaft. Diesen Sicherheitsabstand kann man mit dem englischen Begriff „Slack“ bezeichnen, der hier also einen Spielraum zwischen maximal möglichem und tatsächlich realisiertem Material- und Energiedurchsatz 


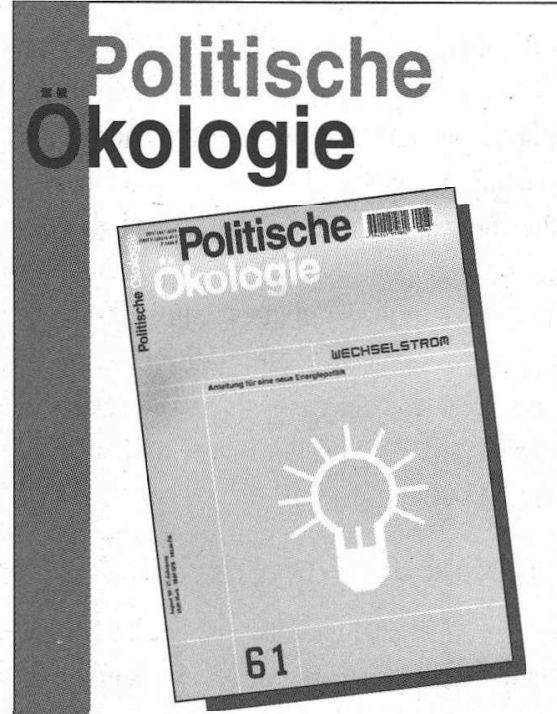

\section{Wechselstrom}

Anleitung für eine neue Energiepolitik

Politische Ökologie Nr. 61

Alle reden über den Ausstieg aus der Atompolitik. Wie aber kommen wir zum Einstieg in eine wirklich veränderte Energiepolitik? Die aktuelle Ausgabe der Politischen Ökologie versucht diesen langen und oftmals auch dornigen Weg zu dokumentieren. Autoren aus Industrie und Forschung diskutieren kontroverse Energiewendeszenarien und die Entwicklungsmöglichkeiten einer „anderen“ Energiepolitik. Potenziale und Perspektiven die sich aufgrund des neuen Energiewirtschaftsgesetztes in Theorie und Praxis ergeben haben sind genauso ein Thema wie konkrete Projekte und Intitiativen. Denn trotz der nach wir vor nicht unerheblichen Hemmnisse gibt es durch aus Anzeichen für einen Wandel.

K.F. Kassel: Einführung in das Thema • J. Radkau: Die Lehren aus der Geschichte - L. Mez: Strategien der deutschen Stromwirtschaft - S. Hentrich: Wirtschaftliche und Politische Wirtschaftshemmnisse - R. Edinger: Potenziale und Barrieren der Photovoltaik - F. Vahrenholt: Trendwende für Photovoltaik $\bullet$ W. Bußmann: Tiefenenergie - K.-D. Clausnitzer: Chancen und Erfahrungen von Energieagenturen $\bullet \mathrm{M}$. Collins: Die Schönauer Energieinitiative -W Roos: Das Durchleitungsgebot

Ausführliche Inhaltsangaben aller Ausgaben finden Sie auch auf unserer Internetseite: www.oekom.de

Jetzt für : pan adress

DM 19,80 Semmelweisstr. 8

direkt zu 82152 Planegg bestellen bei: Fax: 089/85709131 bedeutet, eine Form der Redundanz. Die Schaffung eines Slack trägt dem Umstand Rechnung, das im Kontext der Zielsetzung einer nachhaltigen Entwicklung ,das 'schwächste Glied der Kette' die Leitlinie für die Ausrichtung des Projektes oder der Maßnahme vorgibt" (7). Hier liegt die Logik des Scale- bzw. Steady-State-Ansatzes: Es geht nicht darum, durch umweltpolitische Steuerungsversuche an der als plausibel angenommenen Grenze haarscharf entlang zu fahren, sondern die Makrogröße „Material- und Energiedurchsatz" auf ein Niveau zu bringen, das genug Puffer bietet. Ein solcher Sicherheitsabstand ähnelt dem, was bei Ciriacy-Wantrup ,safe minimum standard" genannt wird (8). Es geht um die Vermeidung irreversibler Schäden, und in der Schadensvermeidung liegt auch der Sinn des Steady-State-Ansatzes.

\section{Jenseits von Daly: Offene Fragen}

Für die (kurzfristigen) Erfolgsaussichten der Steady-State-Orientierung dürfte es von entscheidender Bedeutung sein, inwieweit die Konstanz des Scale Wirtschafts- und also Einkommenswachstum zuläßt oder nicht. Angesichts der erheblichen Redukionserfordernisse und der Logik von Wachstumsprozessen braucht man wohl kein technologischer Pessimist zu sein, um hier Skepsis walten zu lassen. Eine dauerhafte Entkopplung von Wirtschaftsaktivitäten und Umweltverbrauch ist nach dem Stand der Dinge nicht zu erwarten, eine Virtualisierung der Wirtschaft ist trotz Internet und Dienstleistungsorientierung nicht in Sicht. Nicht übersehen werden darf, daß ein ökologischer Strukturwandel - dessen Ziel ja eine sinkende Umweltbelastung ist - Schrumpfungsprozesse impliziert, aber an anderen Stellen der Wirtschaft Wachstum eben nicht ausschließt. Dennoch: Wird die Forderung nach Zukunftsfähigkeit ernst genommen, gehört die Frage des Wirtschaftswachstums ebenso auf die wissenschaftliche und politische Tagesordnung wie die daraus resultierenden Verteilungsprobleme (9). Mindestens ebenso heikel ist die Überlegung, die dem Steady-State-Ansatz in der hier vorgeschlagenen Interpretation zugrundeliegt: Ein Verzicht auf Nutzungsmöglichkeiten zugunsten eines ,Slack“, also die Reduzierung des Material- und Energiedurchsatzes auf ein Niveau, von dem angenommen werden kann, daß es von den ökologischen Tragekapazitäten hinreichend weit entfernt ist. Einem effizienzfixierten ökono- mischen Denken muß dieser Gedanke fremd bleiben (um das mindeste zu sagen). Eine Ökonomik, die Effizienz nicht aus dem Blick verliert, aber den Blick öffnet für Problemlagen und Lösungsmöglichkeiten jenseits von Allokationsfragen, wird sich dieser Herausforderung stellen müssen. Ein enges Verständnis von Wirtschaftlichkeit gegen ökologische Erfordernisse auszuspielen, wird sich nach dem Stand der Dinge als äußerst gefährliche Strategie erweisen.

\section{Anmerkungen}

(1) Daly, Herman E.: Steady State Economics. 2nd Edition with New Essays. Washington, D.C. 1991, S. 17; seine Hervorhebung.

(2) Daly, Herman E.: Steady-State Economics: Concepts, Questions, Policies. GAIA 1 (6), 1992, S. 333.

(3) Daly, Herman E.: Elements of Environmental Macroeconomics. In: Costanza, R.: Ecological Economics. The Science and Management of Sustainability. New York/Oxford 1991, S. 35; seine Hervorhebung.

(4) Sachs, Wolfgang: Ökologischer Wohlstand statt Wachstumsträume. In: Toblacher Gespräche 1994. Ökologischer Wohlstand statt Wachstumsträume. Toblach 1994, S. 18. (5) Hinterberger, Friedrich/ Fred Luks/ Marcus Stewen: Ökologische Wirtschaftspolitik. Zwischen Ökodiktatur und Umweltkatastrophe. Berlin et al. 1996, S. 82.

(6) Vgl. Wynne, Brian: Scientific Knowledge and the Global Environment. In: Redclift, M. / Benton, T. (Hrsg.): Social Theory and the Global Environment. London/New York 1994, S. 183, und

Luks, Fred: Der Steady-State als Grundlage eines Sustainable Development, Dissertation, Hochschule für Wirtschaft und Politik, Hamburg 1998, Kapitel 8.

(7) Kosz, Michael: Ökostevern für eine nachhaltige Entwicklung. Zeitschrift für Umweltpolitik und Umweltrecht 1/1995, S. 24.

(8) Ciriacy-Wantrup, S. V.: Resource Conservation. Economics and Politics. Berkeley/Los Angeles 1952.

(9) Luks, Fred/ Marcus Stewen: Why biophysical assessments will bring distribution issues on top of the agenda. Ecological Economics 29 (1999), S. 33-35.

\section{Der Autor}

Fred Luks isı Mirglied des Forschungsfeldes " 'Globalisierung' und ökologischer Diskurs" an der Hochschule für Wirtschaft und Politik in Hamburg. Zur Zeit ist er an der Vorbereitung der Konferenz "Beyond Growith" beteiligi, die von Heinrich Böll Stiffung, Wuppertal Institui, VÖÖ, ESEE und ISEE organisiert wird. Infos: http://www.wupperinst.org/-

Beyond_Growth/index.html.

Kontakt: Papenhuder Str. 56, 22087 Hamburg,

E-mail: FredLuks@aol.com 
(c) 20I0 Authors; licensee IÖW and oekom verlag. This is an article distributed under the terms of the Creative Commons Attribution Non-Commercial No Derivates License (http://creativecommons.org/licenses/by-nc-nd/3.o/), which permits unrestricted use, distribution, and reproduction in any medium, provided the original work is properly cited. 